\title{
Estimación de la cantidad de potencia suministrada por las celdas fotovoltaicas de un cubesat
}

\section{Estimation of power delivered by photovoltaic cells of a cubesat}

Jesús D. González LloRente

Ingeniero electrónico, especialista en Ingeniería de Software, magíster en Ingeniería Eléctrica, Docente Investigador en la Universidad Sergio Arboleda. Bogotá, Colombia.

Contacto:jesusd.gonzalez@correo.usa.edu.co

\section{Gustavo A. Puerto Leguizamón}

Ingeniero de telecomunicaciones, doctor en Telecomunicaciones, docente de la Universidad Distrital Francisco José de Caldas. Bogotá, Colombia.

Contacto:gapuerto@udistrital.edu.co

Fecha de recepción: 28 de septiembre de 2012

Clasificación del artículo: investigación

Fecha de aceptación: 23 de noviembre de 2013

Financiamiento: Universidad Sergio Arboleda

Palabras clave: celdas fotovoltaicas, CubeSat, órbitas, pico-satélites, potencia.

Key words: CubeSat, orbit, photovoltaic cells, pico-satellites, power.

\section{RESUMEN}

Los CubeSat son pequeños satélites que, debido a las restricciones propias de sus dimensiones, tienen como única fuente de energía celdas fotovoltaicas sobre su superficie. El propósito de este artículo es estimar la cantidad máxima de potencia que puede obtenerse de estas celdas solares, de manera que sea posible realizar un balance de energía cuando se diseñan misiones espaciales con este tipo de satélites. En el escenario analizado, un CubeSat de $10 \mathrm{~cm}$ x $10 \mathrm{~cm}$ x $10 \mathrm{~cm}$ (1U) tiene una cara siempre orientada hacia la Tierra. Se consideran las pérdidas por el coseno del án- gulo y la variación de la temperatura debido a la órbita del satélite. Para la estimación se usa un modelo matemático de las celdas solares, con el cual se calcula el punto de máxima potencia según el ángulo de incidencia de la radiación solar y la temperatura sobre una órbita baja alrededor de la Tierra. Los resultados muestran la variación de la potencia eléctrica máxima suministrada por los paneles sobre la porción iluminada de la órbita, sin considerar el albedo de la Tierra. Además de la potencia, se estiman las curvas corrientevoltaje para diferentes ubicaciones sobre la órbita del CubeSat. 


\section{ABSTRACT}

CubeSats are small satellites that due to their size restriction have photovoltaic cells placed on theirs surface as the only energy source. The purpose of this work is to estimate the maximum amount of power that can be obtained from these solar cells, so that an energy balance can be made during the design of space mission with this kind of satellites. In the analyzed scenario, a CubeSat of $10 \mathrm{~cm}$ $\mathrm{x} 10 \mathrm{~cm} \times 10 \mathrm{~cm}(1 \mathrm{U})$ has always a face oriented toward the Earth. Cosine loss and the temperature variation due to satellite orbit are considered. For the estimation we use a mathematical model of the solar cells, which is also used to estimate the maximum power point according to the incidence angle of the solar radiation and the temperature on a low orbit around the Earth. The results show the variation of the maximum electric power supplied by the panels on the illuminated portion of the orbit, without considering the Earth's albedo. Apart from the power, the current -voltage curves are estimated for different locations over the CubeSat's orbit.

\section{INTRODUCCIÓN}

Un satélite se puede clasificar según su peso; en este contexto se tienen picosatélites que pesan menos de $1 \mathrm{~kg}$, pasando por nanosatélites que tienen un peso entre 1 y $10 \mathrm{~kg}$, minisatélites que pesan entre 100 y $500 \mathrm{~kg}$ hasta satélites estándar y supersatélites con un peso de varias toneladas (Fortescue, Starky Swinerd, 2011). El estándar CubeSat define un satélite en miniatura confinado en un cubo que tiene unas dimensiones de 10x10x10 cm $\mathrm{y}$ un peso que no supera los $1,33 \mathrm{~kg}$. A este cubo se le denomina una unidad (1U) (The CubeSat Program, 2009). El estándar CubeSat fue ideado en 1999 por los profesores Jordi Puig-Suari de la Universidad Politécnica del estado de California (CalPoly) y el profesor Robert Twiggs de la Universidad de Stanford, con el fin de usarlos para realizar investigación espacial de carácter académico o empresarial (Heidt, Puig-Suari, Moore, Nakasukay Twiggs, 2000).

Los CubeSat están compuestos por varios subsistemas conocidos como: manejo de comandos y datos, comunicaciones, energía, orientación y la carga útil (Twiggs y Malphrus, 2011). El subsistema de energía se encarga de suministrar la potencia al resto de subsistemas para su funcionamiento, basado en la captura de energía por medio de paneles solares. Estos paneles están compues-

tos por celdas fotovoltaicas, las cuales convierten la luz solar en electricidad (Ropp, 2007). Las celdas fotovoltaicas para aplicaciones espaciales son fabricadas por Spectrolab y Emcoreen los Estados Unidos, y por AzurSpace en Alemania. Estas celdas son conocidas como III-V, compuesta de una triple juntura de Arseniuro de Galio (GaAs), Fosfato de Indio con Galio (GaInP) y Germanio (Ge), y tienen una eficiencia de alrededor del $30 \%$ para condición en el espacio (Baileyet al., 2009).

Anteriormente se ha determinado la cantidad de potencia solar en la órbita de un picosatélite $\mathrm{Cu}$ beSat, mostrando la variación de la irradiación solar según la traslación de la Tierra (Hernández, Gómez Vargas y Rocha, 2012). En este artículo se estima la cantidad de potencia que puede obtenerse de las celdas fotovoltaicas en las caras de un CubeSat 1U, considerando que el CubeSat tiene una órbita terrestre baja Low Earth Orbit (LEO) y un control de orientación pasivo de manera que una cara está siempre orientada hacia la Tierra. Para la determinación de la cantidad de potencia se usan modelos matemáticos de las celdas fotovoltaicas y se considera el ambiente espacial, considerando la temperatura y la variación de la irradiación solar debido al ángulo de incidencia sobre las celdas. Además, se consideran métodos de extracción de máxima potencia que acoplan la carga al panel. 


\section{investigación}

\section{ESTADO DEL ARTE}

\section{Celdas fotovoltaicas para uso espacial}

Las celdas fotovoltaicas usadas para aplicaciones espaciales y para los CubeSat generalmente son de triple juntura $(\mathrm{GaInP} / \mathrm{GaAs} / \mathrm{Ge})$, con un área de $26,62 \mathrm{~cm}^{2}$, las celdas de Spectrolab y un área de $30,18 \mathrm{~cm}^{2}$ las celdas de Azur Space (Azur Space, 2012; Spectrolab, 2010). Debido a que una cara de un CubeSat 1U tiene un área de $100 \mathrm{~cm}^{2}$, se podrían ubicar máximo dos celdas por cara. Considerando celdas de 28,3\% de eficiencia de Spectrolab, cuyos parámetros tomados de la hoja de datos son listados en la tabla 1 , cada celda suministra $1,04 \mathrm{~W}$ bajo la condición de prueba $\left(1353 \mathrm{~W} / \mathrm{m}^{2}\right.$ y $\left.28^{\circ} \mathrm{C}\right)$; sin embargo, la característica corriente-voltaje y, por tanto, la potencia suministrada varía dependiendo de la temperatura y de la irradiación, como también de la carga que alimentan. Por lo tanto, las dos celdas no siempre suministran 2,08 W.

Para el estudio del comportamiento eléctrico de las celdas fotovoltaicas existen diferentes modelos matemáticos, los cuales pueden estar basados en comportamiento o en circuitos (Gil-Arias y Ortiz-Rivera, 2008). El modelo basado en comportamiento, propuesto inicialmente en (OrtizRivera y Peng, 2005), establece que la relación

Tabla 1. Parámetros de una celda fotovoltaica

\begin{tabular}{|c|c|c|}
\hline Parámetros a $28^{\circ} \mathrm{C}$ y $1353 \mathrm{~W} / \mathrm{m}^{2}$ & Valor & Unidad \\
\hline Voltaje de circuito abierto $\left(\mathrm{V}_{\mathrm{oc}}\right)$ & 2665 & $\mathrm{mV}$ \\
\hline Corriente de corto circuito $\left(\mathrm{I}_{\mathrm{sc}}\right)$ & 464 & $\mathrm{~mA}$ \\
\hline Voltaje de máxima potencia $\left(\mathrm{V}_{\mathrm{mpp}}\right)$ & 2350 & $\mathrm{mV}$ \\
\hline Corriente de máxima potencia $\left(\mathrm{I}_{\mathrm{mpp}}\right)$ & 443,17 & $\mathrm{~mA}$ \\
\hline $\begin{array}{c}\text { Coeficiente de temperatura de } \\
\text { voltaje }\left(\mathrm{TC}_{\mathrm{v}}\right)\end{array}$ & $-5,9$ & $\mathrm{mV} / \mathrm{C}$ \\
\hline $\begin{array}{c}\text { Coeficiente de temperatura de } \\
\text { corriente }\left(\mathrm{TC}_{\mathrm{i}}\right)\end{array}$ & 0,14 & $\mathrm{~mA} / \mathrm{C}$ \\
\hline Ancho de la celda & 39,70 & $\mathrm{~mm}$ \\
\hline Largo de la celda & 69,11 & $\mathrm{~mm}$ \\
\hline
\end{tabular}

Fuente: Tomada de http://www.spectrolab.com (Spectrolab, 2010). entre la corriente $(I)$ y el voltaje $(V)$ de la celda solar está dada por la ecuación (1)

$I=\frac{I_{x}}{1-\exp \left(-\frac{1}{b}\right)}\left[1-\exp \left(\frac{V}{b V_{x}}-\frac{1}{b}\right)\right]$

Donde $V x$ es el voltaje de circuito abierto, $I x$ es la corriente de corto circuito del panel, la constante $b$ es característica del panel y puede calcularse como se describe en (Gil-Arias y Ortiz-Rivera, 2008). Este modelo presenta una gran aproximación a la curva característica de las celdas fotovoltaicas de Spectrolab (Gonzalez-Llorente y Hurtado, 2012). Tanto el voltaje de circuito abierto $V_{x}$ como la corriente de corto circuito $I_{x}$ dependen de la temperatura y la irradiación, y están dados por las ecuaciones (2) y (3)

$$
\begin{gathered}
V_{x}=\left(T-T_{n}\right) T C V+A\left(\exp \left(B \frac{E_{i}}{E_{i n}}\right)-\exp \left(C \frac{E_{i}}{E_{i n}}\right)\right) \\
I_{x}=\frac{E_{i}}{E_{\text {in }}}\left(I_{s c}+\left(T-T_{n}\right) T C I\right)
\end{gathered}
$$

Donde $T$ es la temperatura, $T_{n}$ es la temperatura de prueba especificada en la hoja de datos, $T C v$ es la constante de variación del voltaje con respecto a temperatura, $T C i$ es la constante de variación de la corriente de corto circuito con respecto a la temperatura, $E_{i}$ es la irradiación solar, $E_{\text {in }}$ la irradiación solar estándar para pruebas especificada en la hoja de datos; las constantes $A, B$ y $C$ para la ecuación de circuito abierto se calculan evaluando la ecuación para $V x$ en tres puntos de operación conocidos, como lo describe Gil-Arias (2008).

\section{Orbita sincrónica solar}

La órbita de los CubeSat generalmente es síncrona solar; esto es, el plano orbital rota aproximadamente un grado por cada día para mantenerse orientado hacia el sol, a medida que la tierra gira alrededor de este (Angelo, 2006). Por lo tanto, en algunos puntos de la órbita el satélite recibirá directamente la radiación solar, mientras que en otros puntos estará en la sombra de la tierra. Como consecuencia, la temperatura del CubeSat 


\section{investigación}

variará continuamente al igual que la irradiación; así mismo, la irradiación absorbida dependerá de la orientación hacia el sol.

El periodo orbital, $T_{0}$, puede calcularse a partir de la tercera ley de Kepler, la cual dice que el cuadrado del periodo orbital es proporcional al cubo del eje semimayor, como lo describe la ecuación (4).

$$
T_{0}^{2}=\frac{4 \pi^{2} a^{3}}{\mu}
$$

Donde $a$ es el eje semi-mayor, $\mu$ es la constante gravitacional del planeta (para la Tierra $\mu=3,986$ x $10^{5} \mathrm{~km}^{3} / \mathrm{s}^{2}$ ) (Patel, 2005). Dado que los CubeSat generalmente son puestos en órbitas LEO (de $500 \mathrm{~km}$ a $2000 \mathrm{~km}$ ), aplicando la ecuación (4) el periodo orbital está entre 96,6 minutos y 127,2 minutos. Para el caso del pico-satélite colombiano Libertad 1 con una altura de alrededor de $710 \mathrm{~km}$, su periodo orbital es 99 minutos (Joya, 2007).

\section{Características de irradiación y temperatura en la órbita}

La potencia solar disponible en un sitio es conocida como irradiación y está dada en unidades de vatio por metro cuadrado (W/m²) (Ropp, 2007). Los CubeSat generalmente están ubicados en orbitas LEO, por lo que su distancia a la tierra está entre $500 \mathrm{~km}$ y $2000 \mathrm{~km}$ (Angelo, 2006). La irradiación solar varía con la distancia al sol; la Organización Mundial de Meteorología (World Meteorological Organization [WMO]) estableció una irradiación promedio de $1365 \mathrm{~W} / \mathrm{m}^{2}$ a unidad astronómica (Hyder, Wiley, Halpert, Floody Sabripour, 2000). Debido a los movimientos de traslación de la Tierra y de los satélites en su órbita, la irradiación que recibe un picosatélite varía entre 1328,66 W/m² y 1421,06 W/m² (Hernández et al., 2012). No obstante, la constante solar fuera de la atmosfera terrestre, conocida como Aire Masa Cero (AM0), aceptada por la Sociedad Americana para Prueba y Materiales (American
Society for Testing and Materials [ASTM]), es de $1366,1 \mathrm{~W} / \mathrm{m}^{2}+/-7 \mathrm{~W} / \mathrm{m}^{2}$ (Myers, Emery y Gueymard, 2002), el cual es el valor usado en el presente estudio.

En cuanto a la temperatura de los CubeSat, se han realizado análisis térmicos basados en modelos, los cuales muestran variaciones de $-85^{\circ} \mathrm{C}$ a $50{ }^{\circ} \mathrm{C}$ (Sintes Arroyo, 2009). Además, se cuenta con datos reales enviados por los CubeSat como los del CP3 de la Universidad Politécnica del Estado California (CalPoly), los cuales muestran una variación entre $-30^{\circ} \mathrm{C}$ y $20^{\circ} \mathrm{C}$ (Friedel y McKibbon, 2011). La oscilación de la temperatura es de aproximadamente 90 minutos, lo cual es correspondiente con el periodo estimado anteriormente. El análisis térmico presentado por Erb (2011), muestra una variación de $-32{ }^{\circ} \mathrm{C}$ a $42{ }^{\circ} \mathrm{C}$, lo cual es más coherente con los datos reales del CP3.

\section{METODOLOGÍA}

El procedimiento desarrollado para la determinación de la potencia suministrada por las celdas fotovoltaicas de un CubeSat es presentado en el diagrama mostrado en la figura 1.

Primero se definió el escenario para el CubeSat, que como se especificó anteriormente tiene una órbita LEO sincrónica solar y un sistema de orientación, de manera que una cara se encuentra siempre orientada hacia la Tierra, como se muestra en la figura 2. Se considera una altura de $710 \mathrm{~km}$ y por tanto su periodo orbital es de 99 minutos; aunque la fracción de tiempo que un CubeSat recibe iluminación corresponde al $66,43 \%$, para este trabajo se considera un caso extremo de solo el $50 \%$, esto es 49,5 minutos. Debido al efecto de la irradiación y la temperatura sobre la potencia suministrada por las celdas fotovoltaicas a continuación se describe una aproximación de estas dos variables en el escenario especificado. 


\section{investigación}

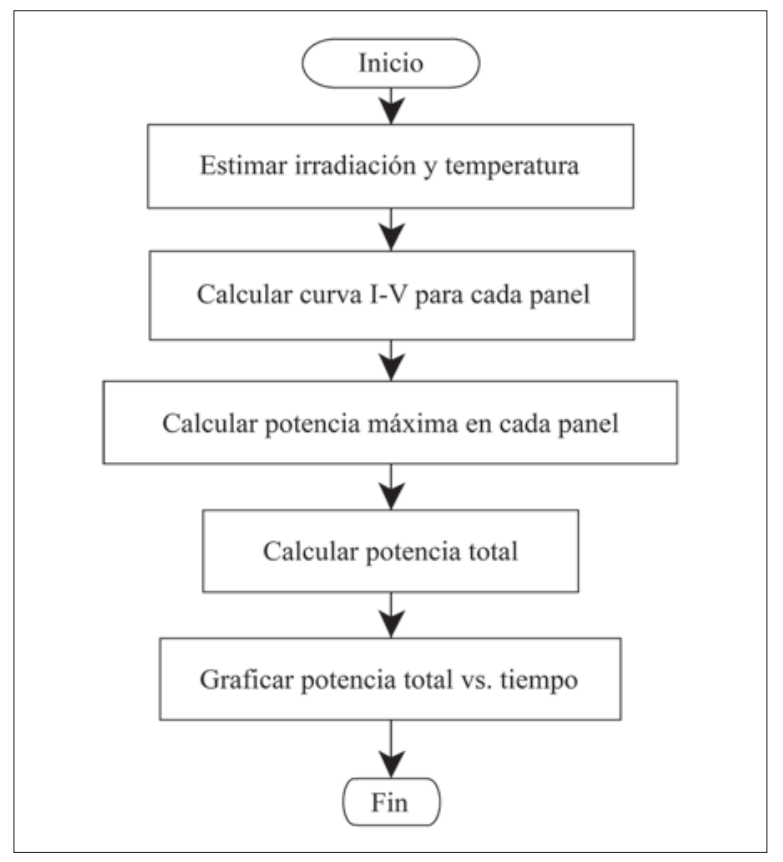

Figura 1. Método para estimar la potencia sobre el CubeSat

Fuente: elaboración propia.

Para este escenario, la cara número 2 del CubeSat siempre está orientada hacia la Tierra, y en el área iluminada las caras 1, 3 y 4 reciben irradiación. En el hemisferio norte se iluminan las caras 1 y 4 , mientras que en el hemisferio sur las caras 3 y 4. El ángulo de incidencia cambia en cada punto de la órbita; en el área iluminada este ángulo va desde $0^{\circ}$ a $180^{\circ}$. Para la cara 1 el área interceptada por la radiación es proporcional al coseno del ángulo $\theta$ entre $0^{\circ}$ y $90^{\circ}$ (Jackson, Moses, Scottiy Blosser, 1984). Para la cara 4 el área interceptada es proporcional al seno del ángulo $\theta$. Por su parte el área interceptada de la cara 3 es proporcional al seno del ángulo $\theta$ entre $90^{\circ}$ y $180^{\circ}$. La radiación absorbida por las celdas solares está directamente relacionada con el área interceptada y es mostrada en la figura 3.

En cuanto a la temperatura, un resultado importante derivado de los datos del CubeSat CP3 del Cal Poly es que no hay diferencia significativa entre la temperatura de los distintos lados del Cube-

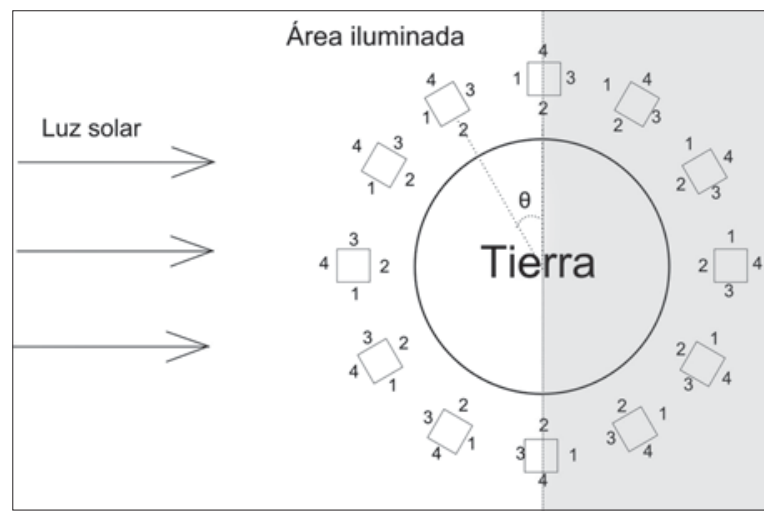

Figura 2. Escenario de un CubeSat $1 \mathrm{U}$ con cara No. 2 hacia la Tierra

Fuente elaboración propia.

Sat (Friedel y McKibbon, 2011). Basado en estos datos y el modelo de temperatura presentado Erb (2011), se propone una representación matemática simplificada para representar la variación de temperatura que permita considerar su efecto en los paneles solares. El aumento en la temperatura mientras recibe irradiación solar está descrita por la ecuación (5), la cual describe una variación de $-32^{\circ} \mathrm{C}$ a $42^{\circ} \mathrm{C}$ mostrada en la figura 4 y similar a la presentada por Erb (2011).

$$
T=74\left(1-e-\frac{t}{0.194}\right)-32,0 \leq t \leq \frac{T}{2}
$$

A partir de los modelos de la variación de la irradiación y la temperatura descritos anteriormente, se determina el voltaje de circuito abierto y la corriente de corto circuito de las celdas fotovoltaicas usando las ecuaciones (2) y (3); con estos valores se obtiene la curva corriente-voltaje usando la ecuación (1) y la potencia máxima con el método descrito anteriormente. Esto es calculado durante la parte iluminada de órbita; es decir durante 49,5 minutos ó 0,82 horas. Para la solución de estas ecuaciones se construyó un modelo en Matlab/Simulink ${ }^{\circledR}$, el cual implementa cada una de las ecuaciones en bloques como lo muestra la figura 5, donde cada uno de los bloques Panel 1, Panel 4 y Panel 3 representa los paneles solares de las caras 1,4 y 3 respectivamente. 


\section{investigación}

\section{RESULTADOS}

Considerando la variación de la temperatura y de la irradiación incidente sobre las caras del CubeSat, la tabla 2 muestra el cambio del voltaje de circuito abierto y de la corriente de corto circuito del panel ubicado en una de las caras, así como también la variación de la potencia máxima. De igual manera la figura 6 muestra la variación de la curva corriente-voltaje para cada uno de las variaciones listadas, mientras que la figura 7 muestra la curva potencia-voltaje para las mismas condiciones.
Para determinar la potencia total obtenida de las tres caras iluminadas del satélite durante la porción iluminada de la órbita se determinó la potencia máxima en cada una de las caras y la suma total es mostrada en la figura 8. Este resultado muestra una potencia máxima de $2,9 \mathrm{~W}$ en 0,17 horas; esto es cuando dos caras reciben la misma irradiación y están sobre el hemisferio norte. Cuando dos caras reciben la misma irradiación sobre el hemisferio sur, los paneles suministran una potencia de $2,75 \mathrm{~W}$. Cuando solo una cara recibe irradiación perpendicular a su superficie la potencia obtenida es $2,31 \mathrm{~W}, 2,06 \mathrm{~W}$ o $2,02 \mathrm{~W}$ según la cara que es iluminada.

Tabla 2. Variación de las condiciones del panel sobre la cara No. 1

\begin{tabular}{|c|c|c|c|c|c|}
\hline Tiempo(h) & Temperatura $\left({ }^{\circ} \mathrm{C}\right)$ & Irradiación $\left(\mathrm{W} / \mathrm{m}^{2}\right)$ & $V_{o c}(\mathrm{~V})$ & $I_{s c}(\mathrm{~mA})$ & Potencia $(\mathrm{W})$ \\
\hline 0,0 & $-32,00$ & 1367,0 & 6,02 & 455,6 & 2,31 \\
\hline 0,1 & $-2,19$ & 1248,8 & 5,60 & 420,0 & 1,98 \\
\hline 0,2 & 15,60 & 914,7 & 5,21 & 309,3 & 1,35 \\
\hline 0,3 & 26,23 & 422,4 & 4,78 & 143,3 & 0,57 \\
\hline
\end{tabular}

Fuente: elaboración propia.

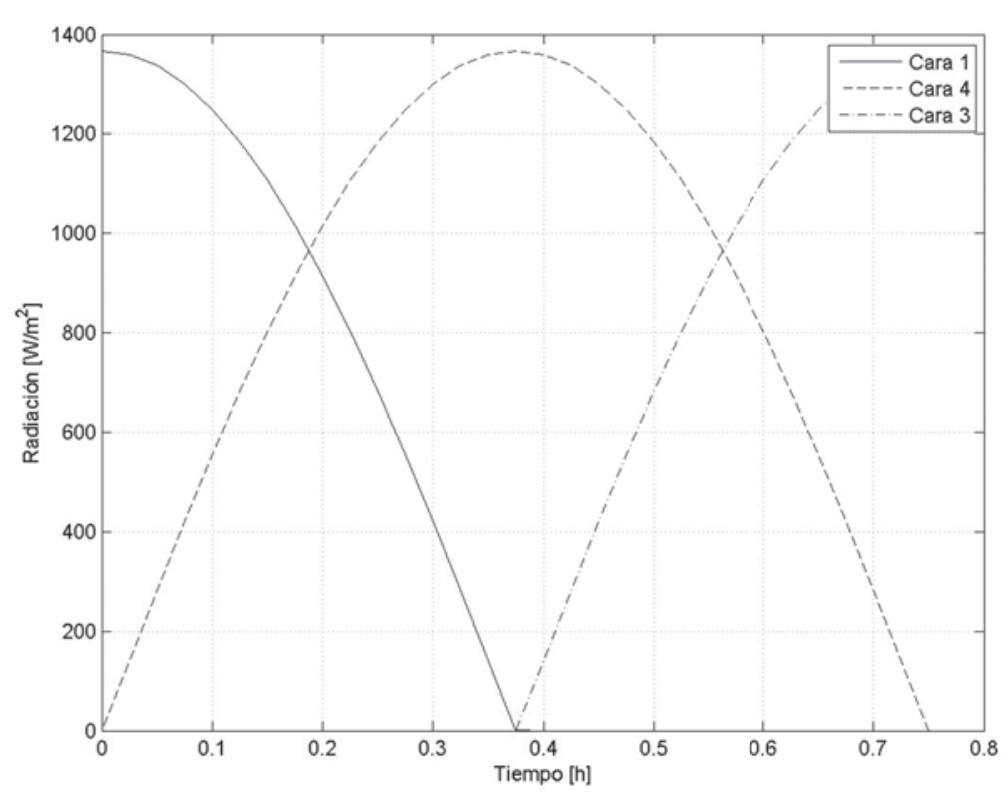

Figura 3. Irradiación solar interceptada por las caras del CubeSat

Fuente: elaboración propia. 


\section{investigación}

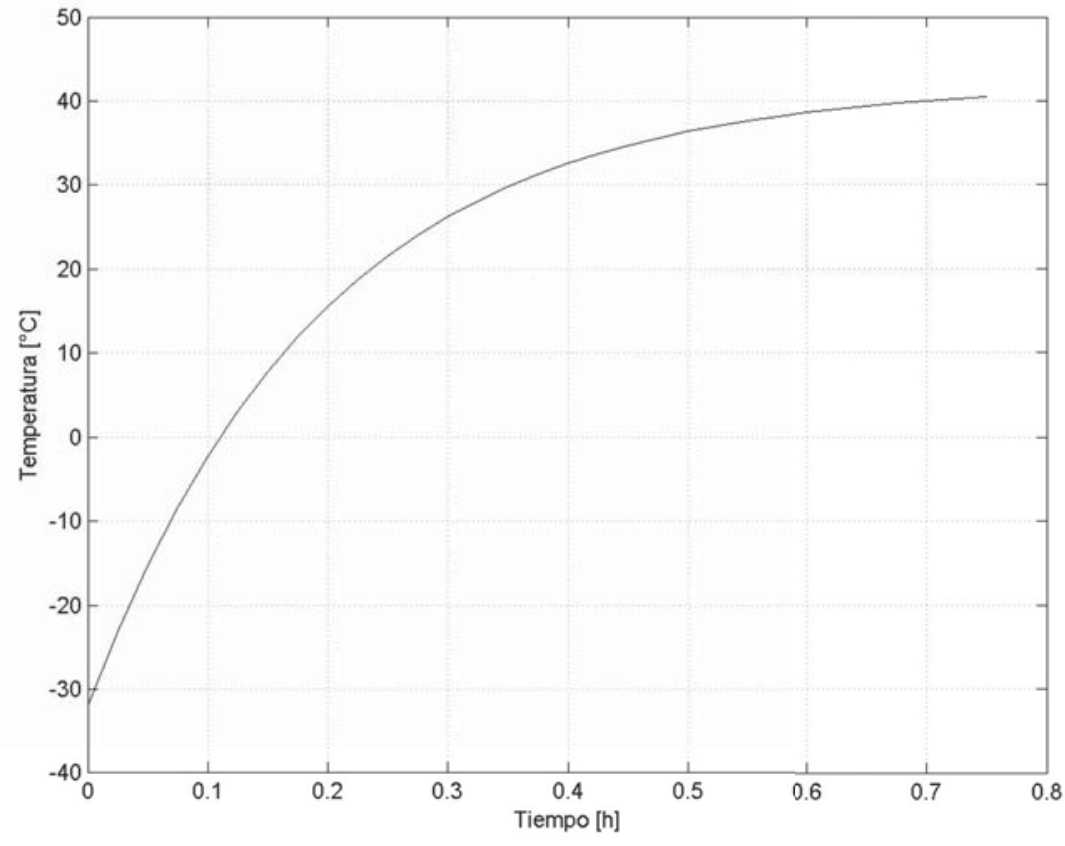

Figura 4. Temperatura del CubeSat durante la parte iluminada de la órbita

Fuente: elaboración propia.

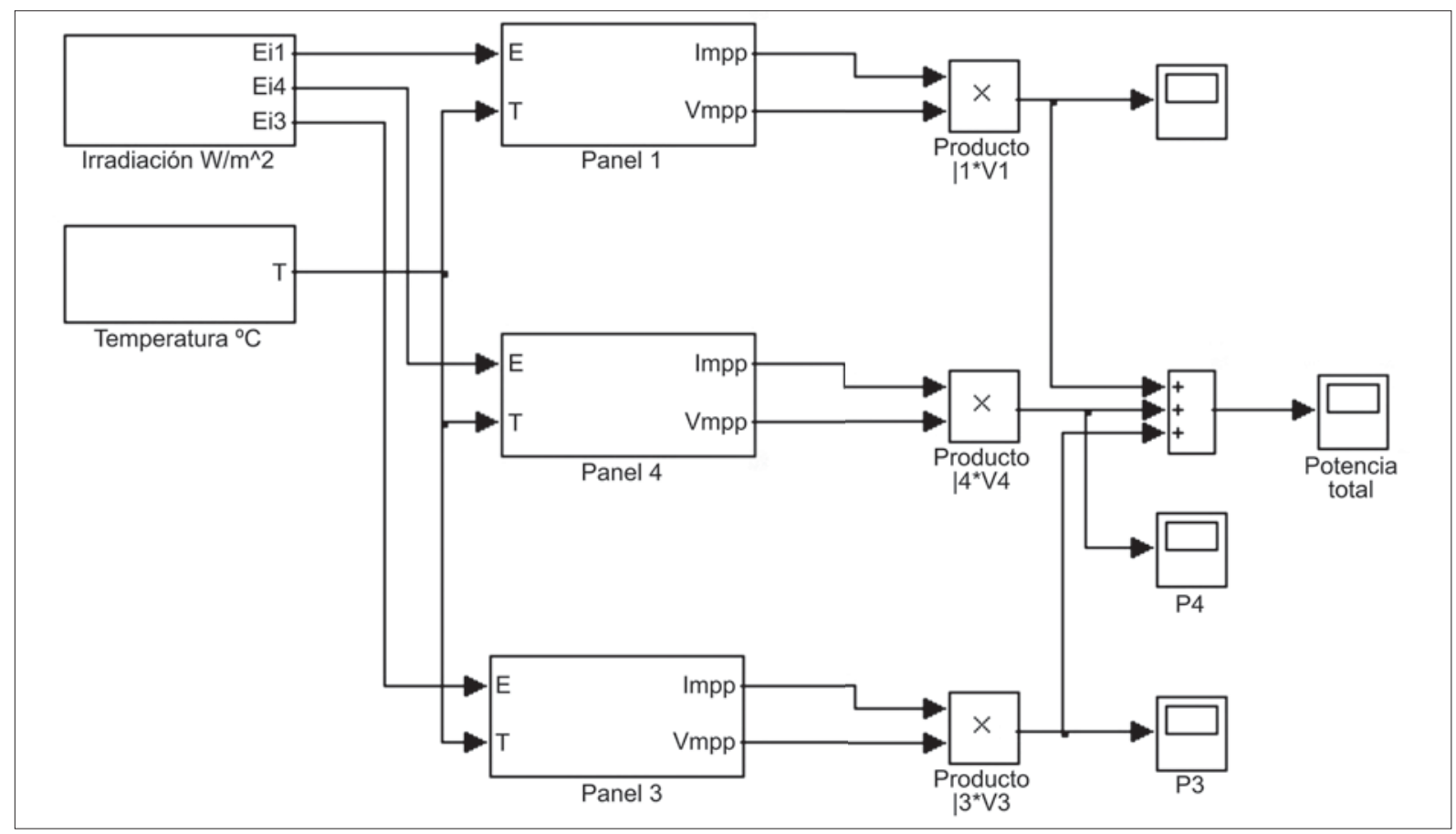

Figura 5. Modelo Matlab/Simulink para estimar la potencia de los paneles de un CubeSat

Fuente: elaboración propia. 


\section{investigación}

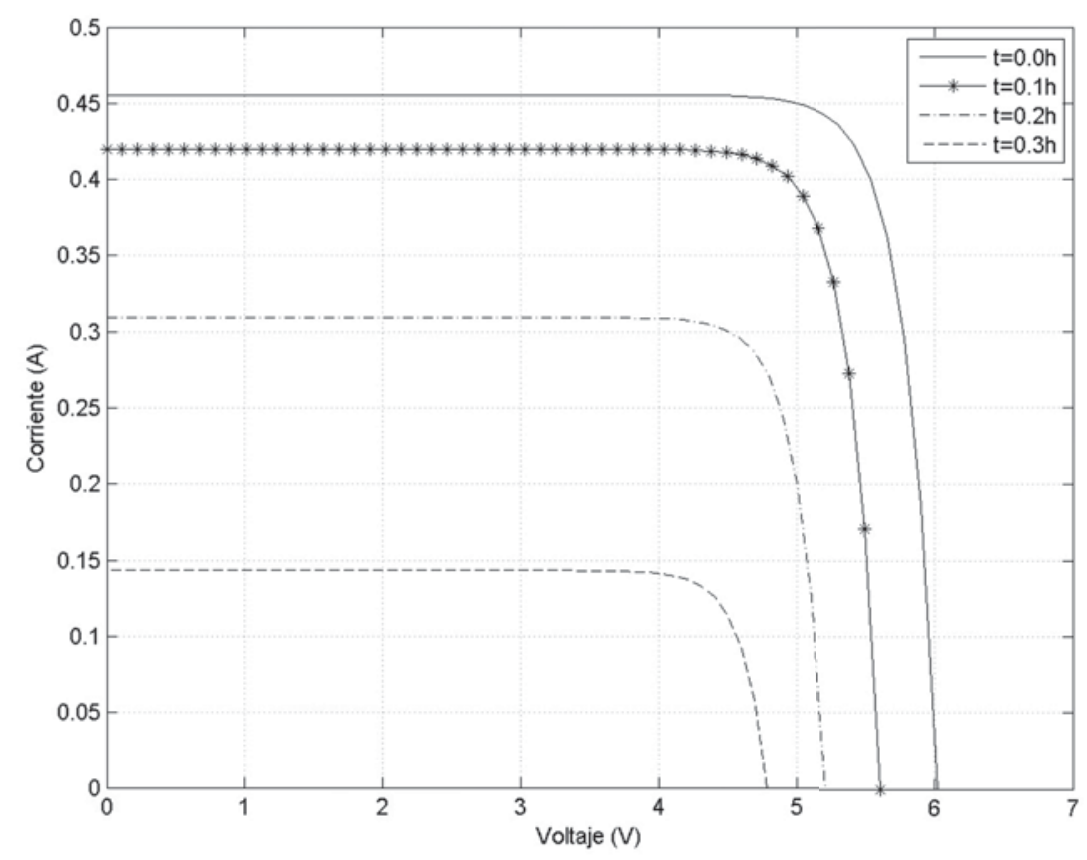

Figura 6. Variación de la curva corriente voltaje sobre el panel de la cara No. 1 Fuente: elaboración propia.

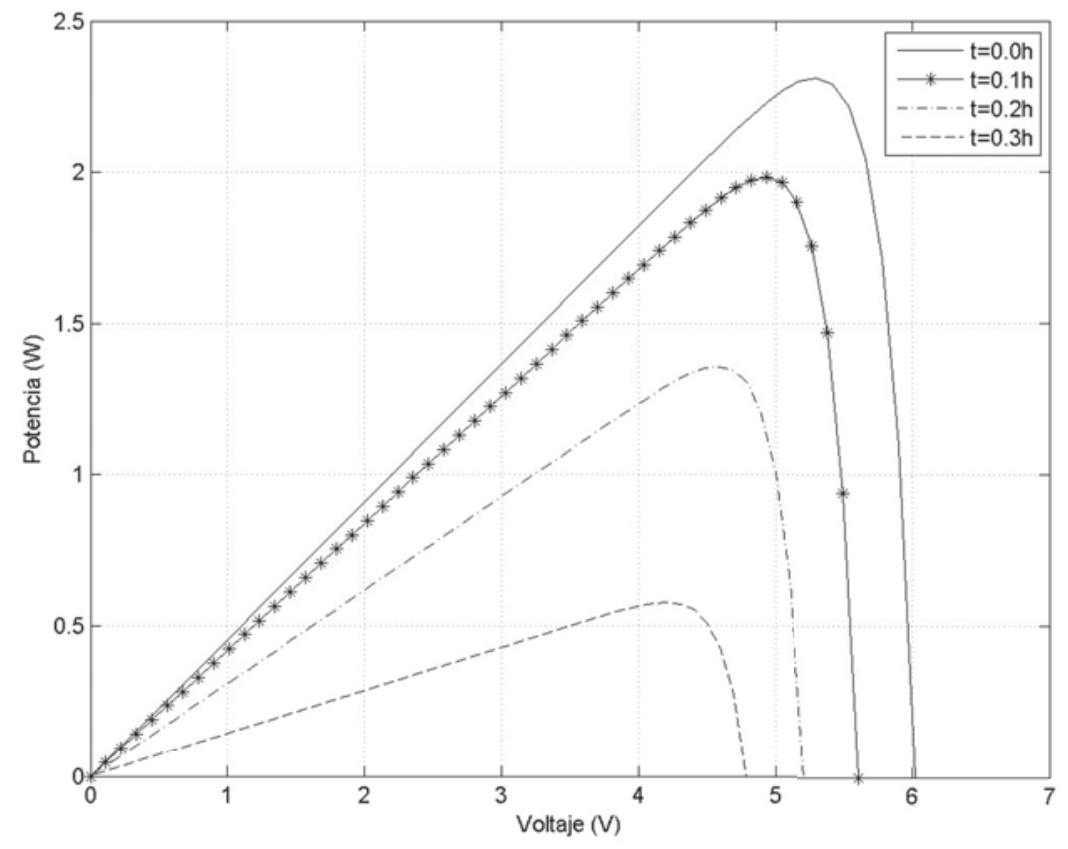

Figura 7. Variación de la curva potencia-voltaje sobre el panel de la cara no.1

Fuente: elaboración propia. 


\section{investigación}

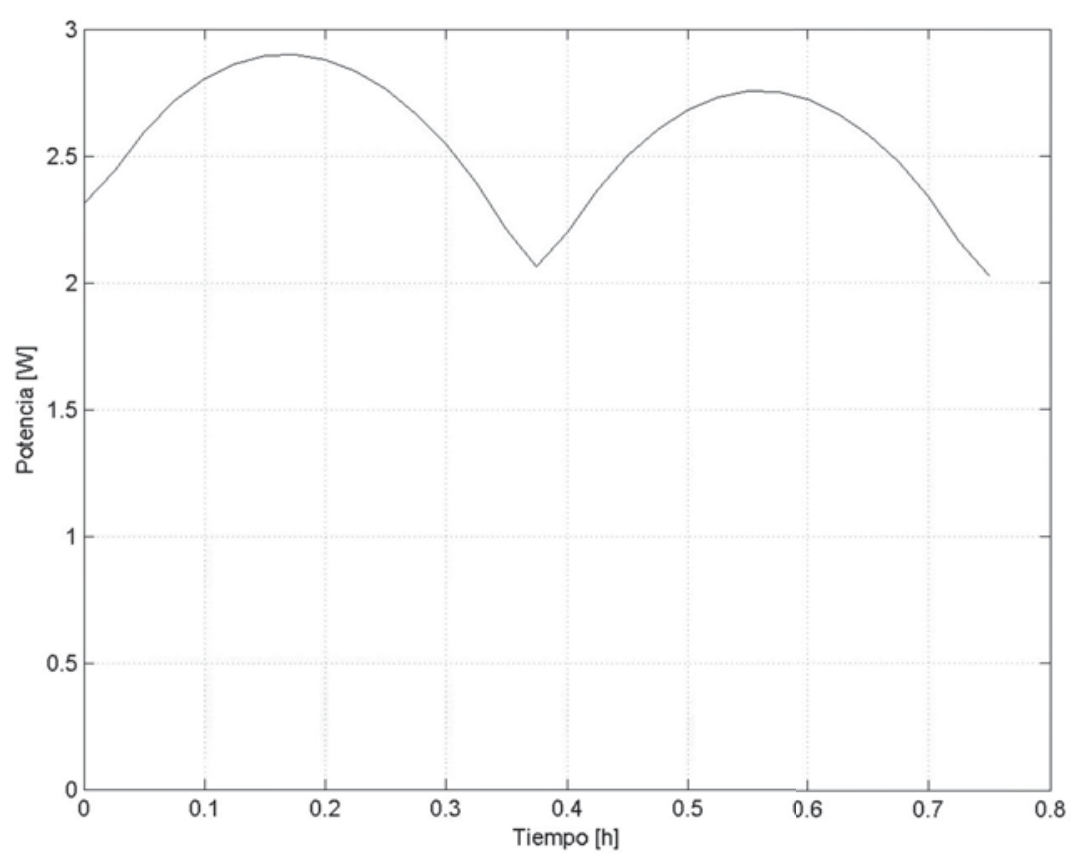

Figura 8. Variación de la potencia máxima sobre la parte iluminada de la órbita del CubeSat

Fuente: elaboración propia.

\section{CONCLUSIONES}

En este trabajo se mostró el uso de modelos de comportamiento de las celdas fotovoltaicas para la estimación de la potencia suministrada por los paneles solares de un CubeSat con una cara orientada hacia la tierra. Se consideró el efecto de la irradiación y la temperatura en la variación del comportamiento de las celdas fotovoltaicas, teniendo en cuenta las pérdidas por la variación del ángulo de incidencia de la radiación solar.

El efecto del cambio del ángulo de incidencia de irradiación sobre los paneles solares afecta considerablemente las curvas corriente-voltaje de las celdas fotovoltaicas, disminuyendo la corriente de corto circuito y, por tanto, la potencia máxima. Sin embargo, la variación de la temperatura afecta en menor escala, disminuyendo la potencia de $2,9 \mathrm{~W}$ a 2,75 W para el mismo ángulo de incidencia al aumentar la temperatura.
Cuando el CubeSat entra a la región iluminada de su órbita, su temperatura se encuentra aproximadamente en $-32{ }^{\circ} \mathrm{C}$ lo cual provoca que un panel entregue una potencia máxima de $2,31 \mathrm{~W}$. La temperatura aumenta debido al efecto de la radiación solar incidente hasta aproximadamente $40^{\circ} \mathrm{C}$; sin embargo, debido a la variación del ángulo de incidencia, la irradiación efectiva absorbida por el CubeSat disminuye desde su valor máximo $1367 \mathrm{~W} / \mathrm{m}^{2}$ hasta cero, lo que provoca la disminución de la potencia máxima que podría suministrar el panel solar. No obstante, el instante en el que dos caras reciben irradiación a un ángulo de $45^{\circ}$ es cuando se obtiene mayor potencia de los paneles solares, la cual es aproximadamente $2,8 \mathrm{~W}$.

\section{FINANCIAMIENTO}

Este resultado de investigación está en el marco del proyecto Análisis para Optimizar el Sistema de Energía de un CubeSat de 3U, código USA- 
2011-0077, perteneciente a la línea de investigación Desarrollo Tecnológico del Grupo de Investigación Esinusa de la Universidad Sergio
Arboleda y financiado con recursos del Fondo de Investigación e Innovación de la Universidad Sergio Arboleda (FIIUSA).

\section{REFERENCIAS}

Angelo, J. ( 2006). Satellites. New York, USA: Facts on File.

Azur Space (2012). 30\% Triple Junction GaAs Solar Cell. Heilbron: AZUR SPACE Solar Power GmbH. Recuperado de http://azurspace.de/index.php?mm=162

Bailey, S., McNatt, J., Raffaelle, R., Hubbard, S., Forbes, D., Fritzenmeier, L. y Maurer, W. (2009). The future of space photovoltaics. 34th IEEE Photovoltaic Specialists Conference (PVSC) Philadelphia, Estados Unidos.

Erb, D. (2011). Evaluating the Effectivenes of Peak Power Tracking Technologies for solar array on small spacecraft (Tesis de maestría). Lexington, Estados Unidos: University of Kentucky.

Fortescue, P., Stark, J. y Swinerd, G. (Eds.). (2011). Spacecraft Systems Engineering. Croydon, UK: John Wiley \& Sons, Ltd.

Friedel, J. y McKibbon, S. (2011). Thermal Analysis of the CubeSat CP3 Satellite. Aerospace Engineering. San Luis Obispo, Estados Unidos: California Polytechnic State University. Recuperado de http://digitalcommons.calpoly.edu/aerosp/46/.

Gil-Arias, O. (2008). Modelado y Simulación de Dispositivos Fotovoltaicos. (Tesis de maestría). Recinto de Mayaguez, Mayagüez, Puerto Rico: Universidad de Puerto Rico.

Gil-Arias, O. y Ortiz-Rivera, E. I. (2008). A general purpose tool for simulating the behav- ior of PV solar cells, modules and arrays. IEEE 11th Workshop on Control and Modeling for Power Electronics, pp. 1-5. Recuperado de: http://ieeexplore.ieee.org/xpl/ articleDetails.jsp?arnumber $=4634686$

Gonzalez-Llorente, J. y Hurtado, R. (2012). Comparación de modelos para celdas solares de alta eficiencia usadas en pequeños satélites y CubeSats. 10th Latin American and $\mathrm{Ca}$ ribbean Conference for Engineering and Technology (LACCEI). Panama.

Heidt, H., Puig-Suari, J., Moore, A. S., Nakasuka, S. y Twiggs, R. J. (2000). CubeSat: A new Generation of Picosatellite for Education and Industry Low-Cost Space Experimentation. 14th Annual/USU Conference on Small Satellites, Estados Unidos.

Hernández, S. R., Gómez Vargas, E. y Rocha, D. F. (2012). Evaluación del desempeño de la radiación emitida por el sol como sistema de suministro de energía a un pico-satélite CUBESAT. Revista Tecnura, 16(31), 19-32.

Hyder, A. K., Wiley, R. L., Halpert, G., Flood, D. J. y Sabripour, S. (2000). Spacecraft Power Technologies. London: Imperial College Press.

Jackson, L. R., Moses, P. L., Scotti, S. J. y Blosser, M. L. (1984). Operational Modules for Space Station Construction. Hampton, VA, USA. Recuperado de http:// ntrs.nasa.gov/archive/nasa/casi.ntrs.nasa. gov/19840013540_1984013540.pdf 


\section{investigación}

Joya, R. A. (2007). Liberta I. Primer satélite colombiano en el espacio. Revista Innovación y Ciencia, 14 (2), 16-23.

Myers, D. R., Emery, K. y Gueymard, C. (2002). Revising and Validating Spectral Irradiance Reference Standards for Photovoltaic Performance. ASES/ASME Solar Energy Conference, Estados Unidos, pp. 367-376.

Ortiz-Rivera, E. I. y Peng, F. Z. (2005). Analytical Model for a Photovoltaic Module using the Electrical Characteristics provided by the Manufacturer Data Sheet. IEEE 36th Conference on Power Electronics Specialists, pp. 2087-2091. Brasil: RECIFE.

Patel, M. (2005). Spacecraft Power System. Boca Raton, Florida, USA: CRC Press.

Ropp, M. (2007). Photovoltaic Systems. Encyclopedia of Energy Engineering and Technology, Estados Unidos: CRC Press.
Sintes Arroyo, P. (2009). Mission and Thermal Analysis of the UPC CubeSat. (Tesis de maestría) Barcelona, España: Universidad Politécnica de Cataluña.

Spectrolab. (2010). 28.3\% Ultra Triple Junction (UTJ) Solar Cells. Sylmar. Estados Unidos. Recuperado de http://www.spectrolab.com/ DataSheets/cells/PV UTJ Cell 5-20-10.pdf

The CubeSat Program. (2009). CubeSat Design Specification rev. 12. Estados Unidos: California Polytechnic State University. Recuperado de http://www.cubesat.org/images/ developers/cds_rev12.pdf

Twiggs, R. y Malphrus, B. (2011). Alternative Spacecraft Designs: CubeSats. Space Mission Engineering The New SMAD. Hawthorne, CA, USA: Space Technology Library. 